\title{
Vacuum instability in external fields
}

\author{
S.P. Gavrilov* and D.M. Gitman ${ }^{\dagger}$ \\ Instituto de Física, Universidade de São Paulo \\ P.O. Box 66318, 05389-970 São Paulo, SP, Brasil
}

(March 2, 2018)

\begin{abstract}
We study particles creation from the vacuum by external electric fields, in particular, by fields, which are acting for a finite time, in the frame of QED in arbitrary space-time dimensions. In all the cases special sets of exact solutions of Dirac equation (IN- and OUT- solutions) are constructed. Using them, characteristics of the effect are calculated. The time and dimensional analysis of the vacuum instability is presented. It is shown that the distributions of particles created by quasiconstant electric fields can be written in a form which has a thermal character and seams to be universal, i.e. is valid for any theory with quasiconstant external fields. Its application, for example, to the particles creation in external constant gravitational field reproduces the Hawking temperature exactly.
\end{abstract}

11.10.Kk, 12.20.Ds, 04.62.+v

Typeset using REVTEX

\footnotetext{
*On leave from Tomsk Pedagogical University, 634041 Tomsk, Russia; present e-mail: gavrilov@snfma1.if.usp.br

†e-mail: gitman@snfma1.if.usp.br
} 


\section{INTRODUCTION}

The effect of particles creation from vacuum by an external field (vacuum instability in an external field) ranks among the most intriguing nonlinear phenomena in quantum theory. Its consideration is theoretically important, since it requires one to go beyond the scope of the perturbation theory, and its experimental observation would verify the validity of the theory in the superstrong field domain. The study of the effect began, in fact, first in 3+1-dimensional QED in connection with the so-called Klein [1] paradox, which revealed the possibility of electron penetration through an arbitrary high barrier formed by an external field. Then in 1951 Schwinger [2] found the vacuum-to-vacuum transition probability in a constant electric field. It became clear that the effect can actually be observed as soon as the external field strength approaches the characteristic value (critical field) $E_{c}=m^{2} c^{3} /|e| \hbar \simeq 1,3 \cdot 10^{16} \mathrm{~V} / \mathrm{cm}$. Although a real possibility of creating such fields under laboratory conditions does not exist now, these fields can play a role in astrophysics, where the characteristic values of electromagnetic fields near and gravitational fields near black holes are enormous. One can also mention that Coulomb fields of superheavy nuclei can create electron-positron pairs. General considerations, concrete calculations and detailed bibliography regarding the vacuum instability in QED can be found in [3] 6]. Particles creation by external gravitational fields [5,7,8] and non-Abelian gauge fields [9] can also be considered in analogy with electrodynamics. There are also various problems in modern quantum theory which are closely related to the vacuum instability in question, for example, phase transitions in field theories, the problem of boundary conditions or topology influence on the vacuum, the problem of consistent vacuum construction in QCD, string theories, multiple particles creation, and so on [5, [7, 10 15].

In spite of the fact that the particles creation effect in external fields was calculated in numerous papers, there are still some problems which are interesting to study and discuss. In the present paper we are going to focus our attention on the time scenario of the process and to consider it in arbitrary dimensions of space-time to be able analyze its dependence 
on the dimension. To fulfill the first part of the program we consider special external fields which act effectively during a finite time and then compare results with ones in a constant field. In fact, such a consideration plays also the role of a regularization and helps to solve divergence problems which appear in constant external fields. The dimensional analysis may be interesting in relation with the study of multidimensional versions of field theories and gravity. Lower dimensions, e.g. $2+1$ dimensions can be of a particular interest. Field theoretical models in such dimensions [16] attracted in the last years a great attention due to various reasons: e.g. nontrivial topological properties, and especially the possibility of the existence of particles with fractional spins and exotic statistics (anyons), having probably applications to fractional Hall effect, high- $T_{c}$ superconductivity and so on [17].

For calculations we are using the general approach, which was elaborated in the framework of the field theory for such kind of problems [18 20,6]. According to this formulation all the information about the processes of particles scattering and creation by an external field (in zeroth order with respect to the radiative corrections) can be extracted from special complete sets of exact solutions of the relativistic wave equations in the external field (INand OUT-solutions). A complete collection of exact solutions of such equations in 3+1 QED is presented in the book [21], in particular, IN- and OUT-solutions and related bibliography can be found in [6]. That is why in the beginning we analyze and classify exact solutions of the Dirac equation in uniform external electric fields in arbitrary space-time dimensions. INand OUT-solutions are presented explicitly for T-constant, adiabatic, and constant electric fields. Probabilities of particles scattering, pairs creation, vacuum-to-vacuum probability and mean numbers of particles created are calculated in arbitrary dimensions for three types of electric fields mentioned above. The full consideration in the case of the T-constant field, which is most important for the time analysis, has no $3+1$-dimensional analog and is presented explicitly for the first time. In spite of some of the formulas in two other cases have $3+1$-dimensional analog, their d-dimensional generalization appears to be non-trivial. Moreover, some of these formulas were not presented even in $3+1$-dimensional case, for example, the total mean numbers of particles created and vacuum-to-vacuum probability in 
the adiabatic field.

Using general expressions obtained for electric fields, which act for a finite time, we study the particle creation effect at small and big times. Thus, in particular, we can estimate a stabilization time of the process, the time of a pair formation, and give a quasiclassical interpretation of a pair creation. Besides, this analysis allows one to select and estimate consistently time divergences, which appear in the constant fields. Comparing results obtained in different time configurations of the electric field, we estimate the role of switching on and off effects in the vacuum instability.

We analyze how the effect of the vacuum instability depends on the space dimensions, on the possible boundary conditions, and on a non trivial topology.

We consider the possibility to add an uniform magnetic field to the electric one and calculate the effect. It turns out that one can formulate universal rules to generalize all the formulas obtained in the pure electric field to the case when the magnetic field is included as well. Its influence on the vacuum instability is studied.

Finally, it is shown, taking into account the vacuum level shift, that the distributions of particles created by the quasiconstant electric fields can be written in a form, which has a thermal character and seams to be universal, i.e. is valid for any theory with a quasiconstant external fields. Its application, for example, to the particles creation in external constant gravitational field, reproduces the Hawking temperature exactly.

\section{GENERAL CONSIDERATION IN AN UNIFORM ELECTRIC FIELD}

The $d$-dimensional Dirac equation in an external electromagnetic field with potentials $A_{\mu}(x)$ has the form (further $\hbar=c=1$ )

$$
\left(P_{\mu} \gamma^{\mu}-m\right) \psi(x)=0, \quad P_{\mu}=i \partial_{\mu}-e A_{\mu}(x)
$$

where $\psi(x)$ is a $2^{\left[\frac{d}{2}\right]}$-component column, $\gamma^{\mu}$ are $\gamma$-matrices in $d$ dimensions 222,

$$
\left[\gamma^{\mu}, \gamma^{\nu}\right]_{+}=2 \eta^{\mu \nu}, \quad \eta^{\mu \nu}=\operatorname{diag}(\underbrace{1,-1,-1, \ldots}_{d}), \quad d=D+1
$$


and $x=\left(x^{\mu}\right)=\left(x^{0}, \mathbf{x}\right), \mathbf{x}=\left(x^{i}\right), \quad \mu=0,1, \ldots, D, \quad i=1, \ldots, D$.

As usual, it is convenient to present $\psi(x)$ in the form

$$
\psi(x)=\left(P_{\mu} \gamma^{\mu}+m\right) \phi(x)
$$

Then the functions $\phi$ have to obey the squared Dirac equation in $d$ dimensions,

$$
\left(P^{2}-m^{2}-\frac{e}{2} \sigma^{\mu \nu} F_{\mu \nu}\right) \phi(x)=0, \quad F_{\mu \nu}=\partial_{\mu} A_{\nu}-\partial_{\nu} A_{\mu}, \sigma^{\mu \nu}=\frac{i}{2}\left[\gamma^{\mu}, \gamma^{\nu}\right]
$$

Let us consider the field $F_{\mu \nu}$ with only one nonzero invariant $I=\frac{1}{2} F_{\mu \nu} F^{\mu \nu}$, which supposes to be negative, $I<0$. In this case there exists a reference frame where only the components $F_{0 i}$ of the field differ from zero. That corresponds to a pure electric field, which is a particular case of external fields, violating the vacuum stability (creating particles). Let this electric field be uniform. It can be nonstationary, but with a constant direction in the space. Then one can always direct it along the axis $x^{D}$. Thus,

$$
F_{0 i}=\left(0, \ldots, 0, E\left(x^{0}\right)\right), F_{i k}=0
$$

For such a field we will use the following potentials: $A_{0}=A_{1}=\ldots=A_{D-1}=0, \quad A_{D}=$ $A_{D}\left(x^{0}\right)$. The constant uniform electric field is of a special interest, because QED with such external field (as with any free external field) can be considered as exact QED (without external fields) with some special initial states of the electromagnetic field [23, 24, 6], which provide the corresponding nonzero mean values of the electromagnetic field. Sometimes an alternating electric field can also be treated as a slight nonuniform free field, which is stipulated by some specific external conditions: existence of a waveguide [25], interference of two coherent waves [26] and so on. However, the study of the constant field shows the existence of divergences related to the infinite action time of the field. More correct consideration demands a regularization in time. For instance, one can consider a field, which acts only a finite time T, being constant within this interval. Such an approach allows also to avoid problems with the definition of IN- and OUT-states in non-switching external fields at $x^{0} \rightarrow \pm \infty$. Another possibility is to consider an alternating field, which 
switches on and off adiabatically at $x^{0} \rightarrow \pm \infty$, and is quasiconstant at finite times. In the next section we are going to consider all the possibilities mentioned to study the time scenario of the particles creation.

Solutions of the equation (3) in the field (4) can be written in the form

$$
\phi_{\mathbf{p}, s, r}(x)=\phi_{\mathbf{p}, s}\left(x^{0}\right) \exp \{i \mathbf{p x}\} v_{s,\{r\}}, \quad r=\left(r_{1}, \ldots, r_{\left[\frac{d}{2}\right]-1}\right), s= \pm 1, \quad r_{j}= \pm 1
$$

where $v_{s,\{r\}}$ are some constant orthonormal spinors, $v_{s,\{r\}}^{\dagger} v_{s,\left\{r^{\prime}\right\}}=\delta_{r, r^{\prime}}$. The eq.(3) allows one to subject these spinors to some supplementary conditions,

$$
\begin{aligned}
& S_{ \pm} v_{\mp 1,\{r\}}=0, S_{ \pm}=\frac{1}{2}\left(1 \pm \gamma^{0} \gamma^{D}\right), \operatorname{rank} S_{ \pm}=J_{(d)}=2^{\left[\frac{d}{2}\right]-1} \\
& R_{ \pm} v_{s,\{\mp 1, \bar{r}\}}=0, \quad R_{ \pm}=\frac{1}{2}\left(1 \pm \frac{i \gamma \mathbf{p}_{\perp}}{\left|\mathbf{p}_{\perp}\right|}\right), \quad \operatorname{rank} R_{ \pm}=\frac{1}{2} J_{(d)}, \quad \text { if } d>3 \\
& \bar{r}=\left(r_{2}, \ldots, r_{\left[\frac{d}{2}\right]-1}\right), \quad p_{\perp}^{a}=p^{a}, a=1, \ldots, D-1, p_{\perp}^{D}=0 .
\end{aligned}
$$

If $d \leq 3$ the quantum numbers $r$ do not appear and for $d=2$ the perpendicular components of the momenta are absent.

Taking into account the conditions (6), one can write an equation for the functions $\phi_{\mathbf{p}, s}\left(x^{0}\right)$

$$
\left[\frac{d^{2}}{d x_{0}^{2}}+\left(p_{D}-e A_{D}\left(x^{0}\right)\right)^{2}+\mathbf{p}_{\perp}^{2}+m^{2}+i s e E\left(x^{0}\right)\right] \phi_{\mathbf{p}, s}\left(x^{0}\right)=0
$$

A formal transition to the spinless case, which corresponds to the use of the Klein-Gordon equation instead of the Dirac one, can be done by putting $s=0$ in (7) and $v_{s,\{r\}}=1$ in (5).

The eq.(7) has two independent solutions at fixed $\mathbf{p}$ and $s$. Thus, an additional quantum number $\zeta$ appears, $\zeta= \pm$. Combining two independent solutions, which correspond to different $\zeta$, one can construct two complete sets of solutions ${ }_{\zeta} \phi_{\mathbf{p}, s}\left(x^{0}\right)$ and ${ }^{\zeta} \phi_{\mathbf{p}, s}\left(x^{0}\right)$, obeying the following conditions

$$
\begin{aligned}
& i \frac{d}{d x^{0}} \zeta \phi_{\mathbf{p}, s}\left(x^{0}\right)={ }_{\zeta} \mathcal{E}_{\mathbf{p} \zeta} \phi_{\mathbf{p}, s}\left(x^{0}\right), \quad \operatorname{sign}{ }_{\zeta} \mathcal{E}_{\mathbf{p}}=\zeta, \quad x^{0} \rightarrow-\infty, \\
& i \frac{d}{d x^{0}}{ }^{\zeta} \phi_{\mathbf{p}, s}\left(x^{0}\right)={ }^{\zeta} \mathcal{E}_{\mathbf{p}}{ }^{\zeta} \phi_{\mathbf{p}, s}\left(x^{0}\right), \quad \operatorname{sign}{ }^{\zeta} \mathcal{E}_{\mathbf{p}}=\zeta, \quad x^{0} \rightarrow+\infty
\end{aligned}
$$

They provide in turn the following behavior 


$$
\begin{aligned}
& H_{o . p .}\left(x^{0}\right){ }_{\zeta} \psi_{\mathbf{p}, s, r}(x)={ }_{\zeta} \mathcal{E}_{\mathbf{p} \zeta} \psi_{\mathbf{p}, s, r}(x), \quad, \operatorname{sign}{ }_{\zeta} \mathcal{E}_{\mathbf{p}}=\zeta, x^{0} \rightarrow-\infty, \\
& H_{o . p .}\left(x^{0}\right){ }^{\zeta} \psi_{\mathbf{p}, s, r}(x)={ }^{\zeta} \mathcal{E}_{\mathbf{p}}{ }^{\zeta} \psi_{\mathbf{p}, s, r}(x), \operatorname{sign}{ }^{\zeta} \mathcal{E}_{\mathbf{p}}=\zeta, \quad x^{0} \rightarrow+\infty,
\end{aligned}
$$

of the corresponding Dirac equation solutions, ${ }_{\zeta} \psi_{\mathbf{p}, s, r}(x)=(\gamma P+m){ }_{\zeta} \phi_{\mathbf{p}, s, r}(x)$ and ${ }^{\zeta} \psi_{\mathbf{p}, s, r}(x)=(\gamma P+m){ }^{\zeta} \phi_{\mathbf{p}, s, r}(x)$. In the eq. (9) $H_{o . p .}=\gamma^{0}(m+\gamma \mathbf{P})$ is one-particle Dirac Hamiltonian, and $\mathcal{E}$ are quasi-energies. The solutions ${ }_{ \pm} \psi_{\mathbf{p}, s, r}(x)$ describe particle $(+)$ and antiparticle (-) in the initial time-instant whereas ${ }^{ \pm} \psi_{\mathbf{p}, s, r}(x)$ describe particle $(+)$ and antiparticle (-) in the final time-instant [19,6].

One can see that the solutions with different $s$ and fixed $\zeta, \mathbf{p}, r$ are dependent. For example,

$$
{ }_{\zeta} \psi_{\mathbf{p}, s, r}(x)=\frac{m+i b_{\mathbf{p}, r}}{{ }_{\zeta} a_{\mathbf{p},-s}} \psi_{\mathbf{p},-s, r}(x)
$$

where $b_{\mathbf{p}, r}=r_{1}\left|\mathbf{p}_{\perp}\right|$ if $d>3, b_{\mathbf{p}, r}=p_{1}$ if $d=3, b_{\mathbf{p}, r}=0$ if $d=2$, and ${ }_{\zeta} a_{\mathbf{p}, s}$ are some coefficients. To see how (10) appears one can use (6), (7), (8) and the following consequence of two latter,

$$
\left[i \frac{d}{d x^{0}}+s\left(p_{D}-e A_{D}\left(x^{0}\right)\right)\right]{ }_{\zeta} \phi_{\mathbf{p}, s}\left(x^{0}\right)={ }_{\zeta} a_{\mathbf{p}, s} \zeta \phi_{\mathbf{p},-s}\left(x^{0}\right), \quad{ }_{\zeta} a_{\mathbf{p},-1 \zeta} a_{\mathbf{p},+1}=m^{2}+\mathbf{p}_{\perp}^{2} .
$$

Similar relation holds for ${ }^{\zeta} \psi_{\mathbf{p}, s, r}(x)$. The eq.(10) means, in fact, that the spin projections of a particle $(+)$ and an antiparticle $(-)$ can take on only $J_{(d)}$ values. Taking that into account, one can only use the following independent solutions,

$$
{ }_{ \pm} \psi_{\mathbf{p}, r}(x)=(\gamma P+m)_{ \pm} \phi_{\mathbf{p}, \pm 1, r}(x), \quad{ }^{ \pm} \psi_{\mathbf{p}, r}(x)=(\gamma P+m)^{ \pm} \phi_{\mathbf{p}, \mp 1, r}(x)
$$

Further, we are going to calculate different matrix elements between the solutions (11) by means of the conventional time independent Dirac scalar product $\left(\psi, \psi^{\prime}\right)=\int \bar{\psi}(x) \gamma^{0} \psi^{\prime}(x) d \mathbf{x}$. In the case under consideration, due to the above mentioned properties (6),(㣙) of the spinors ${ }_{\zeta} \phi_{\mathbf{p}, s, r}(x)$ and ${ }^{\zeta} \phi_{\mathbf{p}, s, r}(x)$, the scalar product can be reduced to a form which is convenient for calculation and, in particular, does not contain $\gamma$ matrices at all,

$$
\left({ }_{+}^{-} \psi_{\mathbf{p}, r,{ }_{+}} \psi_{\mathbf{p}^{\prime}, r^{\prime}}\right)=i(2 \pi)^{D} \delta_{r, r^{\prime}} \delta\left(\mathbf{p}-\mathbf{p}^{\prime}\right)_{+}^{-} \phi_{\mathbf{p},+1}^{*}\left(x^{0}\right) \overleftrightarrow{\partial_{0}}\left(i \partial_{0}+p_{D}-e A_{D}\left(x^{0}\right)\right){ }_{+}^{-} \phi_{\mathbf{p},+1}\left(x^{0}\right)
$$




$$
\begin{aligned}
& \left({ }_{-}^{+} \psi_{\mathbf{p}, r},{ }_{-}^{+} \psi_{\mathbf{p}^{\prime}, r^{\prime}}\right)=i(2 \pi)^{D} \delta_{r, r^{\prime}} \delta\left(\mathbf{p}-\mathbf{p}^{\prime}\right){ }_{-}^{+} \phi_{\mathbf{p},-1}^{*}\left(x^{0}\right) \overleftrightarrow{\partial_{0}}\left(i \partial_{0}-p_{D}+e A_{D}\left(x^{0}\right)\right){ }_{-}^{+} \phi_{\mathbf{p},-1}\left(x^{0}\right) \\
& \left({ }_{+}^{-} \psi_{\mathbf{p}, r},{ }_{-}^{+} \psi_{\mathbf{p}^{\prime}, r^{\prime}}\right)=i(2 \pi)^{D} \delta_{r, r^{\prime}} \delta\left(\mathbf{p}-\mathbf{p}^{\prime}\right)\left(m-i b_{\mathbf{p}, r}\right){ }_{+}^{-} \phi_{\mathbf{p},+1}^{*}\left(x^{0}\right) \overleftrightarrow{\partial_{0}}{ }_{-}^{+} \phi_{\mathbf{p},-1}\left(x^{0}\right)
\end{aligned}
$$

where $\overleftrightarrow{\partial_{0}}=\overrightarrow{\partial_{0}}-\overleftarrow{\partial_{0}}$. (The right side of (12) reproduces the corresponding Klein-Gordon scalar product if one puts formally $\zeta\left[i \partial_{0} \pm\left(p_{D}-e A_{D}\left(x^{0}\right)\right)\right]=m-i b=1$.)

One can see from (8) and (12) that the solutions (11) can be normalized to obey the orthonormality relations,

$$
\left({ }_{\zeta} \psi_{\mathbf{p}, r}, \zeta^{\prime} \psi_{\mathbf{p}^{\prime}, r^{\prime}}\right)=\delta_{\zeta, \zeta^{\prime}} \delta_{r, r^{\prime}} \delta\left(\mathbf{p}-\mathbf{p}^{\prime}\right), \quad\left({ }^{\zeta} \psi_{\mathbf{p}, r} \zeta^{\prime} \psi_{\mathbf{p}^{\prime}, r^{\prime}}\right)=\delta_{\zeta, \zeta^{\prime}} \delta_{r, r^{\prime}} \delta\left(\mathbf{p}-\mathbf{p}^{\prime}\right)
$$

Moreover, each set of solutions ${ }_{\zeta} \psi_{\mathbf{p}, r}(x)$ and ${ }^{\zeta} \psi_{\mathbf{p}, r}(x)$ forms a complete system, thus, we are dealing with the so-called IN- and OUT-sets of solutions correspondingly 19,20,6].

Using (12), one can find decomposition coefficients $G\left(\zeta_{\zeta} \zeta^{\prime}\right)$ of the OUT-solutions in the IN-solutions,

$$
{ }^{\zeta} \psi(x)={ }_{+} \psi(x) G\left(\left.{ }_{+}\right|^{\zeta}\right)+{ }_{-} \psi(x) G\left(\left.{ }_{-}\right|^{\zeta}\right)
$$

The matrices $G\left(\left.{ }_{\zeta}\right|^{\prime}\right)$ obey the following relations,

$$
\begin{aligned}
& G\left(\left.{ }_{\zeta}\right|^{+}\right) G\left(\left.{ }_{\zeta}\right|^{+}\right)^{\dagger}+\kappa G\left(\left.{ }_{\zeta}\right|^{-}\right) G\left(\left.{ }_{\zeta}\right|^{-}\right)^{\dagger}=(\zeta \mathbf{I})^{\frac{1-\kappa}{2}}, \\
& G\left(\left.{ }_{+}\right|^{+}\right) G\left(\left.{ }_{+}\right|^{+}\right)^{\dagger}+\kappa G\left(\left.{ }_{+}\right|^{-}\right) G\left(\left.{ }_{-}\right|^{-}\right)^{\dagger}=0
\end{aligned}
$$

where $\mathbf{I}$ is the unit matrix and $\kappa= \pm 1$ for fermions and bosons respectively. Relations (15) can be derived from the conditions (13). Due to eq.(12) we can easily see that the matrices $G\left(\zeta^{\zeta}\right)$ are diagonal,

$$
G\left(\left.{ }_{\zeta}\right|^{\zeta^{\prime}}\right)_{\mathbf{p}, r, \mathbf{p}^{\prime}, r^{\prime}}=\delta_{r, r^{\prime}} \delta\left(\mathbf{p}-\mathbf{p}^{\prime}\right) g\left(\left.\zeta_{\zeta}\right|^{\zeta^{\prime}}\right)
$$

All the information about the processes of particles creation, annihilation, and scattering in an external field (without radiative corrections) one can extract from the matrices $G\left({ }_{\zeta} \zeta^{\zeta^{\prime}}\right)$ because they define a canonical transformation between IN and OUT creation and annihilation operators in the generalized Furry representation [19,20,6], 


$$
\begin{aligned}
& a^{\dagger}(\text { out })=a^{\dagger}(\text { in }) G\left(\left.{ }_{+}\right|^{+}\right)+b(\text { in }) G\left(\left.{ }_{-}\right|^{+}\right), \\
& b(\text { out })=a^{\dagger}(\text { in }) G\left(\left.{ }_{+}\right|^{-}\right)+b(\text { in }) G\left(\left.{ }_{-}\right|^{-}\right) .
\end{aligned}
$$

Here $a_{n}^{\dagger}(i n), b_{n}^{\dagger}(i n), a_{n}(i n), b_{n}(i n)$ are creation and annihilation operators of IN-particles and antiparticles respectively and $a_{n}^{\dagger}($ out $), b_{n}^{\dagger}($ out $), a_{n}($ out $), b_{n}($ out $)$ are ones of OUT-particles and antiparticles, $n$ presents momentum $\mathbf{p}$ and spin projections $r$. For example, the mean numbers of particles created (which are also equal to the numbers of pairs created) by the external field from the IN-vacuum $\mid 0$, in $>$ with a given momentum $\mathbf{p}$ and spin projections $r$ is

$$
N_{\mathbf{p}, r}=<0, \text { in } \mid a_{\mathbf{p}, r}^{\dagger}(\text { out }) a_{\mathbf{p}, r}(\text { out }) \mid 0, \text { in }>=\left|g\left(\left.{ }_{-}\right|^{+}\right)\right|^{2} \text {. }
$$

Here the standard volume regularization was used, so that $\delta\left(\mathbf{p}-\mathbf{p}^{\prime}\right) \rightarrow \delta_{\mathbf{p}, \mathbf{p}^{\prime}}$. The probabilities of a particle scattering and of a pair creation have the following forms respectively

$$
\begin{aligned}
& P(+\mid+)_{\mathbf{p}, r, \mathbf{p}^{\prime}, r^{\prime}}=\mid<0, \text { out } \mid a_{\mathbf{p}, r}(\text { out }) a_{\mathbf{p}^{\prime}, r^{\prime}}^{\dagger}(\text { in }) \mid 0, \text { in }>\left.\right|^{2}=\delta_{r, r^{\prime}} \delta_{\mathbf{p}, \mathbf{p}^{\prime}} \frac{1}{1-\kappa N_{\mathbf{p}, r}} P_{v}, \\
& P(-+\mid 0)_{\mathbf{p}, r, \mathbf{p}^{\prime}, r^{\prime}}=\mid<0, \text { out } \mid b_{\mathbf{p}, r}(\text { out }) a_{\mathbf{p}^{\prime}, r^{\prime}}(\text { out }) \mid 0, \text { in }>\left.\right|^{2}=\delta_{r, r^{\prime}} \delta_{\mathbf{p}, \mathbf{p}^{\prime}} \frac{N_{\mathbf{p}, r}}{1-\kappa N_{\mathbf{p}, r}} P_{v},
\end{aligned}
$$

where $\mid 0$, out $>$ is the OUT vacuum and

$$
P_{v}=\mid<0, \text { out } \mid 0, \text { in }>\left.\right|^{2}=\exp \left\{\kappa \sum_{\mathbf{p}, r} \ln \left(1-\kappa N_{\mathbf{p}, r}\right)\right\},
$$

is the probability for a vacuum to remain a vacuum. The probabilities for an antiparticle scattering and a pair annihilation are described by the same expressions $P(+\mid+)$ and $P(-+$ 10) respectively.

Thus, to be able to calculate the quantities (18)-(21), in the case under consideration, one has to find solutions of the ordinary differential equation (7), which is in fact Schrödinger equation for a linear oscillator with time-dependent frequency. However, one can make some general conclusions, which do not depend on the concrete time dependence of the electric field in eq.(7). First of all, the matrices $G\left(\left.\zeta_{\zeta}\right|^{\prime}\right)$ are diagonal in all the quantum numbers introduced. Second, the quantum numbers $r$ do not enter in the eq.(7) and, due to the 
structure of the scalar product (12), the matrices can depend on $r$ via a phase only. That is why all the probabilities and the mean numbers do not depend on $r$, so that in the fermionic case the total (summed over all $r$ ) probabilities and the mean numbers are $J_{(d)}$ times greater than the correspondent differential quantities. For example, the total number of particles created with a given momentum $\mathbf{p}$ is

$$
N_{\mathbf{p}}=\sum_{r} N_{\mathbf{p}, r}=J_{(d)} N_{\mathbf{p}, r}
$$

Finally, it is clear that due to the structure of the eq. (7) and the scalar product (12) the dimensionality $d$ enter in the differential probabilities and mean values via the combination $\mathbf{p}_{\perp}^{2}$ only.

\section{T-CONSTANT, ADIABATIC, AND CONSTANT ELECTRIC FIELDS}

\section{A. T-constant field}

To analyze the time dependence of the particles creation effects let us consider the field (匹) with $E\left(x^{0}\right)$ having the form

$$
E\left(x^{0}\right)= \begin{cases}0, & x^{0} \in I \\ E, & x^{0} \in I I \\ 0, & x^{0} \in I I I,\end{cases}
$$

where the time intervals are: $I=\left(-\infty, t_{1}\right), I I=\left[t_{1}, t_{2}\right], I I I=\left(t_{2},+\infty\right), t_{2}-t_{1}=T, t_{2}=$ $-t_{1}$, and $e E>0$ is chosen. Thus, in fact, we consider a constant electric field $E$, which is

acting a finite time T. Further we will call it $T$-constant field. The corresponding potential $A_{D}\left(x^{0}\right)$ can be chosen in the form

$$
A_{D}\left(x^{0}\right)= \begin{cases}E t_{1}, & x^{0} \in I \\ E x^{0}, & x^{0} \in I I \\ E t_{2}, & x^{0} \in I I I .\end{cases}
$$


In each interval $I, I I, I I I$ the equation (7) has two independent solutions, which are correspondingly in $I: \exp \left\{-i p_{0}\left(t_{1}\right) x^{0}\right\}$ and $\exp \left\{+i p_{0}\left(t_{1}\right) x^{0}\right\}$, in $I I: D_{\nu-\frac{1+s}{2}}[(1-i) \xi]$ and $D_{-\nu-\frac{1-s}{2}}[(1+i) \xi]$, and in $I I I: \exp \left\{-i p_{0}\left(t_{2}\right) x^{0}\right\}$ and $\exp \left\{+i p_{0}\left(t_{2}\right) x^{0}\right\}$, where $D_{\nu}(z)$ are Weber parabolic cylinder functions (WPC-functions) [27], and

$$
\nu=\frac{i \lambda}{2}, \quad \lambda=\frac{m^{2}+\mathbf{p}_{\perp}^{2}}{e E}, \quad \xi\left(x^{0}\right)=\frac{e E x^{0}-p_{D}}{\sqrt{e E}}, \quad p_{0}\left(x^{0}\right)=\sqrt{m^{2}+\mathbf{p}_{\perp}^{2}+\left(p_{D}-e A_{D}\left(x^{0}\right)\right)^{2}} .
$$

Using them and conditions (8), one can construct IN- and OUT-solutions ${ }_{-} \psi_{\mathbf{p}, r}(x)$ and ${ }^{+} \psi_{\mathbf{p}, r}(x)$ (see Sect.II). The corresponding expressions for ${ }_{-} \phi_{\mathbf{p},-1}\left(x^{0}\right)$ and ${ }^{+} \phi_{\mathbf{p},-1}\left(x^{0}\right)$ are of the form

$$
\begin{aligned}
& -\phi_{\mathbf{p},-1}\left(x^{0}\right) \\
& =C_{1} \begin{cases}\exp \left\{+i p_{0}\left(t_{1}\right)\left(x^{0}-t_{1}\right)\right\}, & I \\
a_{1} D_{\nu}[(1-i) \xi]+a_{2} D_{-\nu-1}[(1+i) \xi], & I I \\
g\left(\left.{ }^{+}\right|_{-}\right) \exp \left\{-i p_{0}\left(t_{2}\right)\left(x^{0}-t_{2}\right)\right\}+g\left(\left.{ }^{-}\right|_{-}\right) \exp \left\{+i p_{0}\left(t_{2}\right)\left(x^{0}-t_{2}\right)\right\}, & I I I ;\end{cases} \\
& { }^{+} \phi_{\mathbf{p},-1}\left(x^{0}\right) \\
& =C_{2} \begin{cases}g\left(+\left.\right|^{+}\right) \exp \left\{-i p_{0}\left(t_{1}\right)\left(x^{0}-t_{1}\right)\right\}+g\left(\left.{ }_{-}\right|^{+}\right) \exp \left\{+i p_{0}\left(t_{1}\right)\left(x^{0}-t_{1}\right)\right\}, & I \\
a_{1}^{\prime} D_{\nu}[(1-i) \xi]+a_{2}^{\prime} D_{-\nu-1}[(1+i) \xi], & I I \\
\exp \left\{-i p_{0}\left(t_{2}\right)\left(x^{0}-t_{2}\right)\right\}, & I I I,\end{cases}
\end{aligned}
$$

where the normalization constants are

$$
C_{i}=(2 \pi)^{-D / 2}\left(2 p_{0}\left(t_{i}\right) q_{i}\right)^{-1 / 2}, q_{i}=p_{0}\left(t_{i}\right)-(-1)^{i}\left(p_{D}-e A_{D}\left(t_{i}\right)\right), i=1,2
$$

To provide the continuity of the solutions in the time instants $t_{1}$ and $t_{2}$ one has to impose the following conditions

$$
{ }_{-}^{+} \phi_{\mathbf{p},-1}\left(t_{i}+0\right)={ }_{-}^{+} \phi_{\mathbf{p},-1}\left(t_{i}-0\right), \quad \frac{d}{d x^{0}}{ }_{-}^{+} \phi_{\mathbf{p},-1}\left(t_{i}+0\right)=\frac{d}{d x^{0}}{ }_{-}^{+} \phi_{\mathbf{p},-1}\left(t_{i}-0\right),
$$

which allow one to define step by step all the coefficients $a_{i}, a_{i}^{\prime}$, and $g\left(\left.{ }_{ \pm}\right|^{+}\right), g\left(\left.{ }^{ \pm}\right|_{-}\right)$. The first ones are

$$
a_{i}=-(-1)^{i} \frac{p_{0}\left(t_{1}\right) f_{i}^{(+)}\left(t_{1}\right)}{M \sqrt{2 e E}}, \quad a_{i}^{\prime}=(-1)^{i} \frac{p_{0}\left(t_{2}\right) f_{i}^{(-)}\left(t_{2}\right)}{M \sqrt{2 e E}}
$$


where

$$
M=D_{\nu}(z) \frac{d}{d z} D_{-\nu-1}(i z)-D_{-\nu-1}(i z) \frac{d}{d z} D_{\nu}(z)=\exp \left\{-(\nu+1) \frac{i \pi}{2}\right\}
$$

is the Wronskian determinant [27], and

$$
f_{1}^{( \pm)}\left(x^{0}\right)=\left(1 \pm \frac{i \partial_{0}}{p_{0}\left(x^{0}\right)}\right) D_{-\nu-1}[(1+i) \xi], \quad f_{2}^{( \pm)}\left(x^{0}\right)=\left(1 \pm \frac{i \partial_{0}}{p_{0}\left(x^{0}\right)}\right) D_{\nu}[(1-i) \xi]
$$

They can be used to define the latter coefficients. From those we need to know explicitly only $g\left(\left.{ }_{-}\right|^{+}\right)$and $g\left(\left.{ }^{+}\right|_{-}\right)$, which are

$$
\begin{aligned}
& g\left(\left.{ }_{-}\right|^{+}\right)=\exp \left\{(\nu+1) \frac{i \pi}{2}\right\}\left(\frac{p_{0}\left(t_{1}\right) q_{1} p_{0}\left(t_{2}\right)}{8 e E q_{2}}\right)^{\frac{1}{2}}\left[f_{2}^{(-)}\left(t_{2}\right) f_{1}^{(-)}\left(t_{1}\right)-f_{1}^{(-)}\left(t_{2}\right) f_{2}^{(-)}\left(t_{1}\right)\right], \\
& g\left(\left.{ }^{+}\right|_{-}\right)=\exp \left\{(\nu+1) \frac{i \pi}{2}\right\}\left(\frac{p_{0}\left(t_{1}\right) q_{2} p_{0}\left(t_{2}\right)}{8 e E q_{1}}\right)^{\frac{1}{2}}\left[f_{2}^{(+)}\left(t_{2}\right) f_{1}^{(+)}\left(t_{1}\right)-f_{1}^{(+)}\left(t_{2}\right) f_{2}^{(+)}\left(t_{1}\right)\right] .
\end{aligned}
$$

One can see that the coefficients (27) obey the properties

$$
\left.g\left(\left.{ }^{+}\right|_{-}\right)\right|_{p_{D} \rightarrow-p_{D}}=-g\left(\left.{ }_{-}\right|^{+}\right), g\left(\left.{ }^{+}\right|_{-}\right)=g\left(\left.{ }_{-}\right|^{+}\right)^{*}
$$

The first one can be verified directly, whereas the second one is easy to derive comparing representations of the scalar product (12) in the time instants $t_{1}$ and $t_{2}$. Thus, one can conclude that $\left|g\left(\left.{ }_{-}\right|^{+}\right)\right|$is an even function of the momentum $p_{D}$.

To calculate the probabilities and the mean numbers according to the formulas (18-21) we need really to know only the coefficients $g\left(\left.{ }_{-}\right|^{+}\right)$. Comparing (14) and (25), (26), we conclude that the relations (16) hold and we have the expression for the mean numbers of pairs created in the form (18), in which $g\left(\left.{ }_{-}\right|^{+}\right)$is defined by (27). In fact, the mean numbers $N_{\mathbf{p}, r}$ define all the probabilities via the formulas (19)21). As it was shown above this function is even in all the momenta $\mathbf{p}$, including $p_{D}$ and does not depend on the spin quantum number $r$. Using the recipe presented in Sect.II, it is easy to get an explicit form $N_{\mathbf{p}}$ for the the bosonic case from the fermionic one (18) and (27).

Now we are going to analyze the dependence of all the characteristics on the time $T$ and on the momenta. One has to remark that the dependence of the longitudinal momentum $p_{D}$ is of a special interest. This dependence is essentially correlated with the $T$-dependence. 
One can see, e.g. from the four-dimensional case [18,6], that in the constant field $(T=\infty)$ all the characteristics do not depend on the momentum $p_{D}$. This is a source of some kind divergences if one is interested in the total characteristics, which require integration over $p_{D}$. Due to the reasons mentioned above it is enough to analyze only the quantity $N_{\mathbf{p}, r}$. As to the momentum $p_{D}$, one can restrict itself only by $p_{D}$ positive or $p_{D}$ negative. Remark that the momenta $p_{D}$ enter in all the formulas via two dimensionless parameters $\xi_{1}$ and $\xi_{2}$ only,

$$
\xi_{1}=\xi\left(-\frac{T}{2}\right)=\frac{1}{\sqrt{e E}}\left(-e E \frac{T}{2}-p_{D}\right), \xi_{2}=\xi\left(+\frac{T}{2}\right)=\frac{1}{\sqrt{e E}}\left(+e E \frac{T}{2}-p_{D}\right)
$$

which in turn appear in the WPC-functions. Thus, in fact, one needs to analyze the dependence of the latter on $\xi$. It is convenient to consider the region $-\sqrt{e E} \frac{T}{2} \leq \xi_{1}<+\infty, \xi_{2} \geq$ $\sqrt{e E} \frac{T}{2}$, which corresponds to $p_{D}$ negative, $0 \leq-p_{D}<+\infty$, in particular, there always $\xi_{2}>\xi_{1}$.

In the region $\xi_{1} \geq K$, where $K$ is a given number $K>>1+\lambda$ (in terms of the momentum this region corresponds to $\left|p_{D}\right| \geq e E \frac{T}{2}+K \sqrt{e E}$ ) one can use the asymptotic expansion of WPC- functions [27],

$$
D_{\nu}(z)=z^{\nu} \exp \left\{-z^{2} / 4\right\}\left(\sum_{n=0}^{N} \frac{\left(-\frac{1}{2} \nu\right)_{n}\left(\frac{1}{2}-\frac{1}{2} \nu\right)_{n}}{n !\left(-\frac{1}{2} z^{2}\right)^{n}}+O\left(|z|^{-2(N+1)}\right)\right),|\arg z|<\frac{3}{4} \pi,
$$

to conclude that at any $T$ the behavior of the mean numbers (18), (27) is

$$
N_{\mathbf{p}, r}=O\left(\left[\frac{\lambda}{\xi_{1}^{2}}\right]^{3}\right)
$$

For small $T, T<<\frac{1}{\sqrt{e E}}$, and $\left|p_{D}\right|<<e E \frac{T}{2}$, one can get

$$
N_{\mathbf{p}, r}=\frac{e E T^{2}}{4 \lambda+e E T^{2}}[1+O(\sqrt{e E} T)]
$$

At $T<<\frac{\sqrt{\lambda}}{\sqrt{e E}}$ and $T<<\frac{1}{\sqrt{e E \lambda}}$ the form (31) reduces to $N_{\mathbf{p}, r}=e E T^{2} /(4 \lambda)$ and coincides with one which can be derived in the frame of perturbation theory with respect to the external field.

The most important region for the time divergences is one of big $T$, namely, let us consider $T>>\frac{1}{\sqrt{e E}}(1+\lambda)$. In this case $\xi_{2}$ is always big and positive $\xi_{2}>>1+\lambda$, so that the 
asymptotic expansion (29) can be used for any given momentum $p_{D}$. As to the parameter $\xi_{1}$, the whole interval $-\sqrt{e E} \frac{T}{2} \leq \xi_{1}<+\infty$ can be divided in three regions:

$$
\text { a) } \left.-\sqrt{e E} \frac{T}{2} \leq \xi_{1} \leq-K ; \quad b\right)-K<\xi_{1}<K ; \quad \text { c) } \xi_{1} \geq K
$$

The mean numbers $N_{\mathbf{p}, r}$ were estimated in the region c) before, see (30). In the region a) one can use some relations between the WPC-functions [27], for example, $D_{\nu}(z)=$ $\exp \{i \pi \nu\} D_{\nu}(-z)-\frac{\sqrt{2 \pi}}{\Gamma(-\nu)} \exp \frac{i \pi \nu}{2} D_{-\nu-1}(-i z)$, and the asymptotic expansion (29). Then one finds

$$
N_{\mathbf{p}, r}=e^{-\pi \lambda}\left[1+O\left(\left[\frac{1+\lambda}{\xi_{1}}\right]^{3}\right)+O\left(\left[\frac{1+\lambda}{\xi_{2}}\right]^{3}\right)\right], \quad-\sqrt{e E} \frac{T}{2} \leq \xi_{1} \leq-K .
$$

The latter expression allows one to consider the limit $T \rightarrow \infty$ at any given $\mathbf{p}$. In this limit the mean numbers take a simple form

$$
N_{\mathbf{p}, r}=e^{-\pi \lambda}
$$

Thus, when the electric field is acting for a long enough time, the mean numbers of particles created in a given quantum state are stabilized and coincide with expressions which were obtained in the constant electric field in 3+1 QED [18]. (The stabilization (32) was first remarked in 28] for particles created with zero momenta, using a finite action electric field in 3+1 QED.) One can also estimate a characteristic time of such a stabilization. To this end one can see that $\frac{1+\lambda}{\sqrt{e E T}}$ is a small parameter in the decomposition (32) in case $\left|p_{D}\right|<<e E \frac{T}{2}$. If $T>>T_{0}, T_{0}=\frac{1+\lambda}{\sqrt{e E}}$ then the mean numbers are stabilized and $T_{0}$ is the above mentioned characteristic time.

The intermediate region b) does not allows one to use an asymptotic expansion of WPCfunctions to analyze the $\xi_{1}$ dependence. However, one can make some conclusions about its contribution in integrals over the momenta. For example, due to the Fermi statistics $N_{\mathbf{p}, r}$ is always smaller then unity, that is why the integral over the momentum $p_{D}$ in the region b) is less then $2 \sqrt{e E} K$ and is not essential in comparison with the same integral in the region a) at $T \rightarrow \infty$. 
Using these considerations, one can now estimate the sum over the longitudinal momentum $p_{D}$ of $N_{\mathbf{p}, r}$, which is the mean number of particles created with all possible momenta $p_{D}$. To do this we go over to the integral, $\sum_{p_{D}} \rightarrow \frac{L}{2 \pi} \int d p_{D}$, where $L$ is the length in the direction $x^{D}$. As was shown above, at $T>>\frac{1}{\sqrt{e E}}(1+\lambda), N_{\mathbf{p}, r}$ is quasiconstant in the area a), the asymptotics in the area c) has the form (30) and the contribution to the integral form the area b) is less then $\sqrt{e E} K L / \pi$. Then one can conclude

$$
N_{\mathbf{p}_{\perp}, r}=\frac{L}{2 \pi} \int_{-\infty}^{+\infty} N_{\mathbf{p}, r} d p_{D}=\frac{\sqrt{e E} L}{2 \pi}\left[\sqrt{e E} T e^{-\pi \lambda}+O(K)\right] .
$$

Thus, when $T>>\frac{1}{\sqrt{e E}} K>>\frac{1}{\sqrt{e E}}(1+\lambda)$ we can effectively replace the integral over $p_{D}$ by $e E T$ and write

$$
N_{\mathbf{p}_{\perp}, r}=\Delta_{\text {long }} e^{-\pi \lambda}, \quad \Delta_{\text {long }}=\frac{1}{2 \pi} e E L T
$$

The factor $\Delta_{l o n g}$ can be can interpreted as the total number of states with the longitudinal momenta $p_{D}$ of particles created.

It turns out that the expressions for $N_{\mathbf{p}, r}$ and $N_{\mathbf{p}_{\perp}, r}$ at big $T$ for scalar particles coincide with the ones for spinor particles.

To get the total number $N$ of particles created one can sum over the spin projections, using eq.(22), and then over the transversal momenta. The latter sum can be easily transformed into an integral,

$$
N=\sum_{\mathbf{p}} N_{\mathbf{p}}=\frac{V_{(d-1)}}{(2 \pi)^{d-1}} \int d \mathbf{p} N_{\mathbf{p}}
$$

where $V_{(d-1)}$ is (d-1)-dimensional spatial volume. Thus, on gets

$$
N=J_{(d)}^{\frac{1+\kappa}{2}} \frac{V_{(d-1)} T m^{d}}{(2 \pi)^{d-1}}\left(\frac{E}{E_{c}}\right)^{\frac{d}{2}} \exp \left\{-\pi \frac{E_{c}}{E}\right\}, \quad J_{(d)}=2^{\left[\frac{d}{2}\right]-1}
$$

where $E_{c}=m^{2} / e$ is the critical field strength. As one can see the velocity of particles creation is constant at big $\mathrm{T}$. The same result was obtained in $3+1$ dimensions in the framework of the QED at finite temperature, using the functional Schrödinger picture [29]. 
The vacuum-to-vacuum transition probability (21) can be calculated, using both kinds of regularizations, with respect to the volume and to the time. Thus, we get the d-dimensional analog of the well-known Schwinger formula [2],

$$
P_{v}=\exp \{-\mu N\}, \quad \mu=\sum_{n=0}^{\infty} \frac{(-1)^{(1-\kappa) \frac{n}{2}}}{(n+1)^{\frac{d}{2}}} \exp \left\{-n \pi \frac{E_{c}}{E}\right\} .
$$

Returning to the Schwinger result, one ought to say that it was, in fact, obtained from the constant field consideration by means of a regularization. Thus, the space-time volume $V T$ appeared in his formula. One ought to mention the paper [15] where the Schwinger result in the constant electric field was recovered, using the functional Schrödinger equation.

\section{B. Adiabatic field}

Let us consider an alternating uniform electric field (14), where the function $E\left(x^{0}\right)$ has the following form

$$
E\left(x^{0}\right)=E \cosh ^{-2}\left(\frac{x^{0}}{\alpha}\right) .
$$

Such a field switches on and off adiabatically at $x^{0} \rightarrow \pm \infty$, and is quasiconstant at finite times. We will call it adiabatic field. The corresponding nonzero potential is

$$
A_{D}\left(x^{0}\right)=\alpha E \tanh \frac{x^{0}}{\alpha}
$$

In this case solutions of eq.(17) can be written in terms of hypergeometric functions $F(a, b ; c ; y)$ [27, for example,

$$
\begin{aligned}
& { }_{\zeta} \phi_{\mathbf{p},+1}\left(x^{0}\right)={ }_{\zeta} C e^{-i \omega_{-} x^{0}}\left(1+e^{\frac{2 x^{0}}{\alpha}}\right)^{\frac{i \alpha}{2}\left(\omega_{-}-\omega_{+}\right)}{ }_{\zeta} u\left(x^{0}\right), \\
& +u\left(x^{0}\right)=F(a, b ; c ; y), \quad{ }_{-} u\left(x^{0}\right)=y^{1-c} F(a-c+1, b-c+1 ; 2-c ; y), \\
& a=\frac{i \alpha}{2}\left(2 e E \alpha+\omega_{+}-\omega_{-}\right), \quad b=1+\frac{i \alpha}{2}\left(-2 e E \alpha+\omega_{+}-\omega_{-}\right), \\
& c=1-i \alpha \omega_{-}, \quad y=\frac{1}{2}\left(1+\tanh \frac{x^{0}}{\alpha}\right), \quad \omega_{ \pm}=\sqrt{m^{2}+\mathbf{p}_{\perp}^{2}+\left(p_{D} \mp e E \alpha\right)^{2}},
\end{aligned}
$$

where ${ }_{\zeta} C$ are some normalization constants. Considering the asymptotic of the functions (41) at $x^{0} \rightarrow-\infty$, (in this case $F(a, b ; c ; y)=1$ [27]), one can verify that the relations (8) hold and 
${ }_{\zeta} \mathcal{E}_{\mathbf{p}}=\zeta \omega_{-}$. Moreover, the solutions at $x^{0} \rightarrow-\infty$ describe free particles. By analogy one can construct solutions ${ }^{\zeta} \phi_{\mathbf{p},+1}\left(x^{0}\right)$, which describe free particles with energies ${ }^{\zeta} \mathcal{E}_{\mathbf{p}}=\zeta \omega_{+}$ at $x^{0} \rightarrow+\infty$. Their asymptotic form at $x^{0} \rightarrow+\infty$ is ${ }^{\zeta} \phi_{\mathbf{p},+1}\left(x^{0}\right)={ }^{\zeta} C \exp \left(-i \zeta \omega_{+} x^{0}\right)$. To calculate the coefficients $G\left(\left.{ }_{+}\right|^{-}\right)$from (14) it is enough to know the corresponding asymptotic ${ }_{+} \phi_{\mathbf{p},+1}\left(x^{0}\right)$ and ${ }^{-} \phi_{\mathbf{p},+1}\left(x^{0}\right)$, let say at $x^{0} \rightarrow+\infty$, and normalization constants ${ }^{-} C=(2 \pi)^{-D / 2}\left(2 \omega_{+}\left(\omega_{+}-p_{D}+e E \alpha\right)\right)^{-1 / 2}, \quad{ }_{+} C=(2 \pi)^{-D / 2}\left(2 \omega_{-}\left(\omega_{-}+p_{D}+e E \alpha\right)\right)^{-1 / 2}$. Thus, we get the mean numbers of fermions created,

$$
N_{\mathbf{p}, r}=\frac{\sinh \left[\frac{\pi \alpha}{2}\left(2 e E \alpha+\omega_{-}-\omega_{+}\right)\right] \sinh \left[\frac{\pi \alpha}{2}\left(2 e E \alpha+\omega_{+}-\omega_{-}\right)\right]}{\sinh \left(\pi \alpha \omega_{+}\right) \sinh \left(\pi \alpha \omega_{-}\right)} .
$$

In 3+1 QED the corresponding formula was found first in [30]. For scalar particles it has a different form

$$
N_{\mathbf{p}, r}=\frac{\cosh ^{2}\left[\pi \sqrt{\left(e E \alpha^{2}\right)^{2}-\frac{1}{4}}\right]+\sinh ^{2}\left[\frac{\pi \alpha}{2}\left(\omega_{+}-\omega_{-}\right)\right]}{\sinh \left(\pi \alpha \omega_{+}\right) \sinh \left(\pi \alpha \omega_{-}\right)} .
$$

Let us consider the $\alpha$-dependence of these expressions. For small $\alpha, \alpha<<\frac{1}{e E} \sqrt{m^{2}+\mathbf{p}^{2}}$, when the potential changes sharply, we get for fermions

$$
N_{\mathbf{p}, r}=\frac{\left(\pi e E \alpha^{2}\right)^{2}\left(1-\frac{p_{D}^{2}}{m^{2}+\mathbf{p}^{2}}\right)}{\sinh ^{2}\left(\pi \alpha \sqrt{\left.m^{2}+\mathbf{p}^{2}\right)}\right.},
$$

and for bosons

$$
N_{\mathbf{p}, r}=\frac{\left(\pi e E \alpha^{2}\right)^{2}\left[\left(e E \alpha^{2}\right)^{2}+\frac{p_{D}^{2}}{m^{2}+\mathbf{p}^{2}}\right]}{\sinh ^{2}\left(\pi \alpha \sqrt{\left.m^{2}+\mathbf{p}^{2}\right)}\right.} .
$$

Small $\alpha$ in the case under consideration corresponds in a sense to small $T$ of the T-constant field. Thus, we have to compare the expressions (31) and (44). One can see that they are quite different, so that the effects of switching on and off are essential at small times.

Further let us consider big $\alpha$ only, $\alpha>>\frac{1}{\sqrt{e E}}(1+\sqrt{\lambda})$. Then the mean numbers for fermions and bosons have the same form,

$$
N_{\mathbf{p}, r}=\exp \left\{-\pi \alpha\left(\omega_{+}+\omega_{-}-2 e E \alpha\right)\right\}
$$

Let us take small longitudinal momenta $\left|p_{D}\right|<<e E \alpha$, then 


$$
N_{\mathbf{p}, r}=\exp \left\{-\pi \lambda\left[1+\left(\frac{p_{D}}{e E \alpha}\right)^{2}\right]\right\}
$$

Considering the limit $\alpha \rightarrow \infty$, one gets the formula (33). That means that the effects of switching on and off are not essential at big times and small longitudinal momenta. For big longitudinal momenta $\left|p_{D}\right|>>e E \alpha$, the mean numbers of particles created are exponentially small,

$$
N_{\mathbf{p}, r}=\exp \left\{-2 \pi \alpha\left(\left|p_{D}\right|-e E \alpha\right)\right\}
$$

Let us find the total numbers of particles created with all the longitudinal momenta at any fixed $\mathbf{p}_{\perp}, r$. Passing from the summation over $p_{D}$ to the corresponding integration, we get

$$
N_{\mathbf{p}_{\perp}, r}=\frac{L e E \alpha}{2 \pi \sqrt{\lambda}} e^{-\pi \lambda}
$$

Comparison with the formula (35) shows that the adiabatic field at big times (big $\alpha, \alpha>>$

$\frac{1}{\sqrt{e E}}(1+\sqrt{\lambda})$ and fixed $\mathbf{p}_{\perp}, r$ is equivalent to the $T$-constant field at $T>>\frac{1}{\sqrt{e E}}(1+\lambda)$ with the identification $\alpha=\sqrt{\lambda} T$. To do summation over all transversal momenta, it is convenient to use the representation

$$
\frac{1}{\sqrt{\lambda}}=2 \int_{0}^{\infty} \exp \left(-\pi \lambda s^{2}\right) d s .
$$

Then the total number $N$ of the particles created reads

$$
N=J_{(d)}^{\frac{1+\kappa}{2}} \frac{V_{(d-1)} \alpha \delta m^{d}}{(2 \pi)^{d-1}}\left(\frac{E}{E_{c}}\right)^{\frac{d}{2}} \exp \left\{-\pi \frac{E_{c}}{E}\right\}
$$

where

$$
\delta=\int_{0}^{\infty} d t t^{-\frac{1}{2}}(t+1)^{-\frac{d-2}{2}} \exp \left(-t \pi \frac{E_{c}}{E}\right)=\sqrt{\pi} \Psi\left(\frac{1}{2},-\frac{d-2}{2} ; \pi \frac{E_{c}}{E}\right)
$$

is expressed via the confluent hypergeometric function [27. The vacuum-to-vacuum transition probability $P_{v}$ has the form

$$
\begin{aligned}
& P_{v}=\exp \{-\mu N\}, \quad \mu=\sum_{n=0}^{\infty} \frac{(-1)^{(1-\kappa) \frac{n}{2}} \epsilon_{n+1}}{(n+1)^{\frac{d}{2}}} \exp \left\{-n \pi \frac{E_{c}}{E}\right\}, \\
& \epsilon_{n}=\delta^{-1} \sqrt{\pi} \Psi\left(\frac{1}{2},-\frac{d-2}{2} ; n \pi \frac{E_{c}}{E}\right) .
\end{aligned}
$$


If $E / E_{c}<<1$ one can use an asymptotic of $\Psi$-function [27], $\Psi\left(\frac{1}{2},-\frac{d-2}{2} ; n \pi \frac{E_{c}}{E}\right)=\frac{1}{\sqrt{\pi n}} \sqrt{\frac{E}{E_{c}}}+$ $O\left(\left[\frac{E}{E_{c}}\right]^{-3 / 2}\right)$. Then $\delta=\sqrt{\frac{E}{E_{c}}}, \epsilon_{n}=n^{-\frac{1}{2}}$ and $\mu=1$. In this case the adiabatic field is equivalent to $T$-constant field with the identification $\alpha=T \sqrt{\frac{E_{c}}{E}}$. At strong fields $E \sim E_{c}$ all the terms with different $\epsilon_{n}$ contribute to the sum in (51) and the expression for $P_{v}$ differs essentially from the one for the $T$-constant field.

One can also remark that the case of a periodic alternating electric field in $3+1$ dimensions was also considered in the literature, for example, quasiclassically [31], and exactly [32].

\section{Constant field}

Here we consider the case of a constant uniform electric field (4). Then $E\left(x^{0}\right)=E$ and potential $A_{D}=E x^{0}$. Solutions of the eq.(17) in such a field can be found in the form

$$
{ }_{+}^{-} \phi_{\mathbf{p}, s}\left(x^{0}\right)=C D_{\nu-\frac{1+s}{2}}( \pm(1-i) \xi),{ }_{-}^{+} \phi_{\mathbf{p}, s}\left(x^{0}\right)=C D_{-\nu-\frac{1-s}{2}}( \pm(1+i) \xi) .
$$

Using an asymptotic expansion of WPC-functions (29), one can get the asymptotics of the quasi-energies,

$$
{ }_{\zeta} \mathcal{E}_{\mathbf{p}}=\zeta\left|e E x^{0}-p_{D}\right|, \quad{ }^{\zeta} \mathcal{E}_{\mathbf{p}}=\zeta\left(e E x^{0}-p_{D}\right)
$$

so that IN and OUT-solutions can be constructed from (52) by means of eq. (11). The same asymptotic expansion (29) allows one to calculate the normalization constants, $C=$ $(2 \pi)^{-D / 2}(2 e E)^{-1 / 2} \exp \{-\pi \lambda / 8\}$ for spinor case and $C=(2 \pi)^{-D / 2}(2 e E)^{-1 / 4} \exp \{-\pi \lambda / 8\}$

for scalar one. Straightforward calculations, similar to ones where made in the two previous cases, lead to the expression (33) for the mean numbers of particles created. It does not depend on the dimensionality of the space and coincides with the result which was derived in [18] for 3+1 QED. In that paper the authors used quasiclassical considerations to advocate the classification of the solutions (52). The constant character of the field does not allow one to treat consistently time divergences, so that they got over them "by hand", using also quasiclassical considerations. Now one can see that the consideration of the $T$-constant field 
gives a possibility both to ground all the results obtained from the constant field solutions, solving consistently the problem of the time divergences and to go beyond the scope of the constant field to analyze the time scenario of the process.

\section{Inclusion of a magnetic field}

In the same manner as before one can consider a more general case when a constant uniform magnetic field is included, provided the invariant $\mathrm{I}$ is negative. In fact, in $d>3$ there are $\left[\frac{d}{2}\right]-1$ independent invariant parameters $H_{j}, j=1,2, \ldots, H_{\left[\frac{d}{2}\right]-1}$ of the magnetic field, that corresponds to the possibility to construct $\left[\frac{d}{2}\right]$ invariants of the electromagnetic field. In a convenient reference frame, the magnetic part of the field tensor $F_{\mu \nu}$ is presented by the components $F_{\mu \nu}^{\perp}=\sum_{j=1}^{\left[\frac{d}{2}\right]-1} H_{j}\left(\delta_{\mu}^{j+1} \delta_{\nu}^{j}-\delta_{\nu}^{j+1} \delta_{\mu}^{j}\right)$. One can always select solutions of the squared Dirac equation (3) as eigenfunctions for all independent nonzero terms, which describe the interaction of intrinsic magnetic moment of a particle with the external magnetic

field. In this case the matrices $G\left(\left.{ }_{\zeta}\right|^{\prime}\right)$ are diagonal and one can construct them using the corresponding expressions in the pure electric field. Namely, for $d>3$ one has to make there a replacement

$$
\begin{aligned}
& \left|\mathbf{p}_{\perp}\right|^{2} \rightarrow \sum_{j=1}^{\left[\frac{d}{2}\right]-1} \omega_{j}+\omega_{0}, \quad \omega_{0}= \begin{cases}0, & \mathrm{~d} \text { is even } \\
p_{d-2}^{2}, & \mathrm{~d} \text { is odd },\end{cases} \\
& \omega_{j}=\left\{\begin{array}{ll}
\left|e H_{j}\right|\left(2 n_{j}+1-r_{j}\right), n_{j}=0,1, \ldots, & H_{j} \neq 0 \\
p_{j}^{2}+p_{j+1}^{2}, & H_{j}=0
\end{array} .\right.
\end{aligned}
$$

In the presence of the magnetic field some momenta $p_{j}$ have to be replaced by the discrete quantum numbers $n_{j}$. The number of these momenta $p_{j}$ corresponds to the number of nonzero parameters $H_{j}$. The magnetic field lifts the degeneracy in spin projections in all the characteristics of the particles creation effect.

We present here explicit formulas in presence of the magnetic field for the total characteristics $N$ and $P_{v}$ in case of the $T$-constant field at big $T$, 


$$
\begin{aligned}
& N=J_{(d)}^{\frac{1+\kappa}{2}} \frac{V_{(d-1)} T m^{2} \beta(1)}{2^{(d-1)} \pi^{d / 2}} \frac{E}{E_{c}} \exp \left\{-\pi \frac{E_{c}}{E}\right\} \\
& P_{v}=\exp \{-\mu N\}, \quad \mu=\sum_{n=0}^{\infty} \frac{(-1)^{(1-\kappa) \frac{n}{2}} \beta(n+1)}{(n+1) \beta(1)} \exp \left\{-n \pi \frac{E_{c}}{E}\right\},
\end{aligned}
$$

where

$$
\begin{aligned}
& \beta(n)=\prod_{j=1}^{(d-2) / 2}\left\{\frac{e H_{j}}{\sinh \left(n \pi H_{j} / E\right)}\left[\cosh \left(n \pi H_{j} / E\right)\right]^{\frac{1+\kappa}{2}}\right\}, d \text { is even }, \\
& \beta(n)=\left(\frac{m^{2} E}{n \pi E_{c}}\right)^{\frac{1}{2}} \prod_{j=1}^{(d-3) / 2}\left\{\frac{e H_{j}}{\sinh \left(n \pi H_{j} / E\right)}\left[\cosh \left(n \pi H_{j} / E\right)\right]^{\frac{1+\kappa}{2}}\right\}, d \text { is odd } .
\end{aligned}
$$

The corresponding formulas for $3+1$-dimensional case were first written in [18,3], and, in fact, can be derived easily from the calculations of Schwinger [2]. They follow from (54) at $d=4$

There exists a possibility to get also exact results for the particles creation in case when a plane wave is added to the combination of electric and magnetic fields. The corresponding calculations for $(3+1)$-dimensional case were made in cited6 for the electric field plus a plane wave field, and in 34 for a general combination of electric, magnetic, and plane wave fields. They can be generalized to any dimensions, combining the approaches of [34 and of the present paper.

\section{DISCUSSION}

\section{A. Time and space-dimensional analysis}

The calculation and analysis presented in Sect. III for fields, which are effectively acting

for a finite time, allows one to study both pairs formation in time and the role of switching on and off effects. Besides, due to the fact that these calculations are made in arbitrary dimensions of the Minkowski space-time, one gets a possibility to analyze the influence of the dimensionality on the vacuum instability.

Studying the T-constant field, one can see that the stabilization of the mean numbers of particles created with given $\mathbf{p}, r$ in the form (33) for the longitudinal momenta $\left|p_{D}\right|<<e E \frac{T}{2}$ 
comes at $T>>T_{0}$, where $T_{0}=\frac{1}{\sqrt{e E}}(1+\lambda)$. The characteristic time $T_{0}$ can be called stabilization time. At the same time $N_{\mathbf{p}, r}$ for the big longitudinal momenta $\left|p_{D}\right|>>e E \frac{T}{2}$ decrease according to the power low (30).

The stabilization of the mean numbers with given $\mathbf{p}, r$ in the adiabatic field in the same form (33) comes for the longitudinal momenta $\left|p_{D}\right|<<e E \alpha$ at $\alpha>>\alpha_{0}, \alpha_{0}=\frac{1}{\sqrt{e E}}(1+\sqrt{\lambda})$. For big $\left|p_{D}\right|>>e E \alpha$ the mean numbers are exponentially small (48). For big $\alpha$ the adiabatic field varies slowly and coincides nearly with the constant one in the time interval $\left|x^{0}\right| \leq \alpha$. Then $\alpha_{0}$ is a characteristic time of the stabilization in this field. Thus, the stabilization time $\alpha_{0}$ in the adiabatic field differs from the corresponding time $T_{0}$ in the T-constant field. Thus, one can believe that the stabilization process depends of the switching on and off effects. In the case $E / E_{c}<1$, which corresponds to $\lambda>1$, one can see the stabilization comes quicker for adiabatic field than for the T-constant one $\left(\alpha_{0}<T_{0}\right)$, i.e. the adiabatic form of switching on and off affects less the quantum system than the instantaneous one in the T-constant field. If $E / E_{c} \geq 1$, there exists a domain of the transversal momenta $\mathbf{p}_{\perp}$ where $\lambda \leq 1$. In this case the stabilization times in both cases are the same, $\alpha_{0} \sim T_{0} \sim \frac{1}{\sqrt{e E}}$, so that for any $E$ the relation $\alpha_{0} \leq T_{0}$ holds.

Thus, one can conclude, that in some cases T-constant and adiabatic electric fields act on the vacuum in a similar way. However, the momentum dependence of the mean numbers $N_{\mathbf{p}, r}$ differs essentially at big momenta for both fields. That is related to the switching on and off effects. To estimate the role of the effects of switching on and off on the whole it is convenient to compare total characteristics. First, let us compare the total mean numbers with all the longitudinal momenta, namely, compare the formulas (35) and (49). In this case the effective action of both kind of fields is the same if to identify $\alpha$ with $\sqrt{\lambda} T$. In spite of this identification of $\alpha$ and $T$ is different for different $\lambda$ (for different $\mathbf{p}_{\perp}$ ), one can use it in a domain $\mathbf{p}_{\perp}$ of the transversal momentum, $\left|\Delta \mathbf{p}_{\perp}\right|<<\sqrt{m^{2}+\mathbf{p}_{\perp}^{2}}$. As to the total numbers (37) and (50), they coincide if one accepts the identification $\alpha=T \delta^{-1}$. However, this identification provides only the coincidence of $P_{v}$ for both cases (38) and (51) if $E / E_{c}<<1$ 
(then $\left.\delta=\sqrt{\frac{E}{E_{c}}}\right)$. In this case the coefficients $\mu$ in (38) and (51) are the same. One can conclude that the effects of switching on and off are not essential for $E / E_{c}<<1$ and for big $T>>\frac{1}{m}\left(\frac{E_{c}}{E}\right)^{3 / 2}$, or for big $\alpha$ respectively. In case of strong fields, $E / E_{c} \geq 1$, these effects appear to be essential and one has to take into account the back reaction of particles created for more realistic external field definition (e.g. see [5, 4, 14]).

The stabilization of the mean numbers of particles created with given $\mathbf{p}, r$ at $T>>T_{0}$ can be interpret in the following way: In the T-constant electric field at the finite time instant $\frac{T}{2}$ the pairs are created with quantum numbers $\left|p_{D}\right|<e E \frac{T}{2}$ (equal for particles and antiparticles). This corresponds to the region $0<\pi^{D}\left(\frac{T}{2}\right)<e E T$ of the observed kinetic momenta, $\pi^{D}\left(\frac{T}{2}\right)=-\left(p_{D}-e A_{D}\left(\frac{T}{2}\right)\right)=-p_{D}+e E \frac{T}{2}$ of a particle in each pair (direction of antiparticle kinetic momenta is opposite). At $T>T_{0}$ the effects of switching on and off are already not essential. That is why the probabilities of pairs creation do not depend on the time instants $t,-\frac{T}{2}<t<\frac{T}{2}$. One can think that at this time instant the particles in the pairs are materialized with almost zero longitudinal kinetic momenta at any given $\mathbf{p}_{\perp}$, i.e. with the energies $\sqrt{m^{2}+\mathbf{p}_{\perp}^{2}}$. Then the electric field accelerates them until the end of its action. Let us suppose that a particle was created at a time instant $t$. An expression for the kinetic momentum of such a particle at the final time instant (which is equal to its expression in the time instant when the field switches off) can be found solving the classical equation of motion $\frac{d \pi^{D}}{d x^{0}}=e E$, so that $\pi^{D}\left(\frac{T}{2}\right)=e E\left(\frac{T}{2}-t\right)$. Thus, a particle, which was discovered with the quantum number $p_{D}$ at the final time instant, was created at the time instant $t=\frac{p_{D}}{e E}$. Then the integration over the longitudinal momenta $p_{D}$ is equivalent to one over the time $t, \int d p_{D}=e E T$. This conclusion coincides with one derived in course of the strict quantum analysis presented in Subsect. IIIA. According to the same interpretation, for particles with relatively nonzero mean numbers, the maximum value of the kinetic momentum $\pi^{D}\left(\frac{T}{2}\right)$ is $e E T$ that corresponds to the particles, which were created at the initial time $t=-\frac{T}{2}$, whereas its minimal value is 0 and corresponds to the particles, which were created at the final time instant $t=\frac{T}{2}$ ). This conclusion coincides also with one derived from quantum 
consideration in Subsect. IIIA.

In the conclusion of the time analysis, one can remark that the time $T_{0}$, which was introduced by us as the stabilization time, was interpreted in some papers as the time of a pair creation [18,28]. However, we have seen that in the adiabatic field the stabilization time $\alpha_{0}$ is different, thus $T_{0}$ is not an universal characteristic and depends of the field form. In this connection one can propose another characteristic time, which a pair formation in a quasiconstant electric field. Indeed, as we have mentioned above, all the results in the T-constant and adiabatic fields are comparable if $E / E_{c}<<1$. In this case the adiabatic form of the field is disturbing the quantum system less than the T-constant field. Here $\alpha_{0}<<T_{0}$ and $\alpha_{0} \approx T_{0}^{f}=\frac{\sqrt{\lambda}}{\sqrt{e E}}$. Since the adiabatic field is quasiconstant for the time interval $T_{0}^{f}$ and it is big enough for the stabilization, one can interpret $T_{0}^{f}$ as the time of a pair formation. One can extrapolate this interpretation of $T_{0}^{f}$ for any field strength $E$. A quasiclassical consideration confirms this interpretation. Thus, a virtual particle with initial zero energy gets from the electric field for the time $T_{0}^{f}$ the energy $\sqrt{m^{2}+\mathbf{p}_{\perp}^{2}}$ necessary for the materialization. It is easily to see that the time $T_{0}^{f}$ is always either less than the stabilization times $T_{0}, \alpha_{0}$ or equal to them. Some other consideration related to the time $T_{0}^{f}$ one can meet in the next subsection.

Turning to the dimensional analysis, one can see that the increase of degrees of freedom, due to the increase of the dimensionality of the space-time itself and due to the related increase of the spinning space dimension $J_{(d)}$, affects essentially the total numbers of particles created in the unit of the volume and the probability for vacuum to remain a vacuum. Thus, the increase of spinning degrees of freedom leads to an increase of $N$ and $P_{v}$ at any ratio $E / E_{c}$. In particular, in $d>3$ the numbers of fermions created is greater than the one of bosons. An increase of spatial dimensions leads to a decrease of the total numbers of particles created in the unit of the volume and the probability for a vacuum to remain a vacuum at $E / E_{c}<1$ and their increase at $E / E_{c}>1$.

The presence of walls or of a nontrivial topology affects the spectrum of particles created. If the length $L_{i}$ of the space in the direction of an axis $x^{i}$ is restricted by the walls 
the corresponding momentum $\left|p_{i}\right|=\frac{2 \pi n}{L_{i}}, n=1,2, \ldots \quad$ is quantized. At $L_{i} \sim \frac{1}{m}$ the dependence of the mean numbers on the boundary conditions is essential. At $L_{i}<<\frac{1}{\sqrt{e E}}$ the mean numbers $N_{\mathbf{p}, r}$ in the quasistationary fields are very small for any strength $E$. In this connection one can treat $L_{0}=\frac{1}{m}\left[1+\left(\frac{E_{c}}{E}\right)^{1 / 2}\right]$ as a characteristic dimension of the system, for which the boundary conditions are essential. At $E / E_{c} \geq 1$ it is the Compton wave length. It is interesting to remark that the stabilization times $T_{0}, \alpha_{0}$ coincide with $L_{0}$ at $E / E_{c}=1$.

Imposing periodic conditions in the direction of an axis $x^{i}$ (that corresponds, in particular, to the torus topology), one gets for the momentum $\left|p_{i}\right|=\frac{2 \pi n}{L_{i}}, n=0,1,2, \ldots$. Then at $L_{i}<<\frac{1}{\sqrt{e E}}$ only particles with $p_{i}=0$ can be created. If the electric field has the same direction, then the total number $N$ and the probability $P_{v}$ do not depend on time $T$, since this dependence arises in course of a summation over the longitudinal momenta. It is interesting that the presence of the magnetic field acts as a dimensional reduction. Indeed, in the strong magnetic field with some $H_{j}>>E$ the lowest energy level of a boson can not be less than $\left|e H_{j}\right|$, whereas for a fermion it can. That means that the strong magnetic field acts on bosons as some walls and on fermions as the presence of the torus topology. Thus, one can see that if some of the magnetic fields are strong enough, then the corresponding spin projection become frozen and total characteristics, like total mean numbers decrease. These dimensional effects may be relevant to the matter creation at early universe.

\section{B. Relation between the vacuum instability in external electromagnetic and gravitational fields}

It is interesting to compare particles creation in external electromagnetic fields and in external fields of different nature, for example, in external gravitational fields. To this end one can use results obtained in the quasiconstant electric fields and in the static gravitational fields. The latter problem was considered first by Hawking [8] who, in particular, calculated the mean numbers of particles created by static gravitational field of a black hole with mass $M$ in a specific thermal environment, 


$$
N_{n}=\left[\exp \left\{2 \pi \frac{\omega}{g_{(H)}}\right\}+\kappa\right]^{-1},
$$

where $\omega$ is the energy of a particle created, which supposes to be dependent on a complete set of quantum numbers $n, g_{(H)}=\frac{G M}{r_{g}^{2}}$, where $r_{g}$ is the gravitational radius, so that $g_{(H)}$ is free falling acceleration at this radius. This spectrum was interpreted as a Planck distribution with the temperature $\theta_{(H)}=\frac{g_{(H)}}{2 \pi k_{B}}\left(k_{B}\right.$ is the Boltzmann constant). As before $\kappa=+1$ for fermions and $\kappa=-1$ for bosons. It is also known 35] that an observer, which is moving with a constant acceleration $g_{(R)}$ (with respect to its proper time), will register in the Minkowski vacuum some particles (Rindler particles). The mean numbers of Rindler bosons have the same Planck form (55) (with $\kappa=-1$ ), where one has to replace $g_{(H)}$ by $g_{(R)}$, so that the correspondent temperature is $\theta_{(R)}=\frac{g_{(R)}}{2 \pi k_{B}}$. One can find many other examples when the particles creation in external gravitation fields (and due to a nontrivial topology) can be described by means of an effective temperature [5,7] ( see also references in the recent publications [36]). On the other hand the distributions obtained in external electromagnetic fields have not the thermal form at a first glance. Nevertheless, there were attempts to find close relations between the distributions in both cases, moreover, to derive Hawking distribution from the one in external electromagnetic field.

In [37, the distribution (33) at $\mathbf{p}_{\perp}=0$ was interpreted as the Boltzmann one for particles in the ground state with the energy $m$ and the effective temperature $\theta_{(E)}=\frac{2 e E}{\pi m}$. The same temperature follows from some other consideration [38] for the same restricted case. Unfortunately, such an interpretation does not allow one to include other states with nonzero momenta in the consideration.

In [39, the authors did not introduce an effective temperature directly in the electrodynamical case but tried to find a relation between both distributions, in particular, to extract the Hawking temperature from the electrodynamical formulas. We are going to repeat briefly here this consideration, using some new details, which came from the results of the present paper. As was established, a particle with given momenta is created in a time instant with energy $\omega=\sqrt{m^{2}+\mathbf{p}_{\perp}^{2}}$, which corresponds to zero longitudinal kinetic 
momentum at this time instant. Thus, namely this expression plays the role of the total energy of the particle at the time moment of creation. Then we can compare equations of motion for a classical particle in a constant electric field $d \pi / d x^{0}=e \mathbf{E}$ with ones in the static gravitational field $d \pi / d x^{0}=\omega \mathrm{g}$. In the latter, $\omega$ is the total energy of the test particle and $\mathbf{g}$ is the three-dimensional gravitational field strength vector. Although these equations are formally similar, there is a fundamental difference between them: the electromagnetic coupling constant $e$ of a charged particle is not affected by its motion, while the coupling to the gravitational field is proportional to the total energy of the test particle. The latter property is a direct consequence of the equivalence principle. Let us formally replace the electric field strength $E$ by a quantity that characterizes the gravitational field strength $\mathrm{g}$ and exploit the equivalence principle by considering, that the coupling of the particle to the field is proportional to the energy of the former, which also allows us to replace $e$ by $\omega$. The expression that arises from (33) as a result of these replacements can be interpreted as the mean numbers of particles created by the corresponding gravitational field and have the form of the Boltzmann distribution with characteristic temperature $\theta^{\prime}=\frac{\mathrm{g}}{\pi k_{B}}$. If $\mathrm{g}$ is the gravitational field strength at the horizon of the black hole $g_{(H)}$, then $\theta^{\prime}$ is only 2 time greater than the Hawking temperature $\theta_{(H)}$. In spite of the fact that an explicit progress was achieved in the way of comparing both distributions, some questions remain. For example, why the temperature derived by means of the equivalence principle from the electrodynamical distribution differs by the factor 2 from the Hawking one? Is there a thermal interpretation of the electrodynamical distribution (33) or some universal form of particles creation spectrum which is valid in both cases? Below we propose some interpretation of the electrodynamical formulas which pretends to answer these questions. We go beyond the classical consideration, taking into account properties of the physical vacuum in the time dependent external field.

First, one can remark that due to the time dependence of the potential $A_{D}\left(x^{0}\right)$, which defines the quasiconstant electric field, the level of the vacuum energy changes with time. Thus, one has to calculate carefully the difference between the energies of the system in the 
initial (vacuum) and in the final (with particles) states. Let us do the calculations in the case of fermions and in zero order with respect to radiative corrections in the T-constant electric field. In this case the corresponding Hamiltonian has the form

$$
H\left(x^{0}\right)=\int \bar{\Psi}(x) H_{o . p .} \Psi(x) d \mathbf{x},
$$

where $H_{\text {o.p. }}$ was defined in (9), and $\bar{\Psi}(x), \Psi(x)$ are electron-positron field operators in the generalized Furry picture [19,20,6]. Being written in terms of IN- and OUT- operators of creation and annihilation at $x^{0} \rightarrow \mp \infty$ respectively, the Hamiltonian $H\left(x^{0}\right)$ has the diagonal forms,

$$
\begin{aligned}
& H\left(x^{0}\right)=\sum_{\mathbf{p}, r} p_{0}\left(t_{1}\right)\left[a_{\mathbf{p}, r}^{\dagger}(i n) a_{\mathbf{p}, r}(i n)+b_{\mathbf{p}, r}^{\dagger}(i n) b_{\mathbf{p}, r}(i n)-1\right], \quad x^{0} \rightarrow-\infty, \\
& H\left(x^{0}\right)=\sum_{\mathbf{p}, r} p_{0}\left(t_{2}\right)\left[a_{\mathbf{p}, r}^{\dagger}(\text { out }) a_{\mathbf{p}, r}(\text { out })+b_{\mathbf{p}, r}^{\dagger}(\text { out }) b_{\mathbf{p}, r}(\text { out })-1\right], \quad x^{0} \rightarrow+\infty,
\end{aligned}
$$

where, as before, $t_{2}=-t_{1}=\frac{T}{2}$, and $p_{0}\left(t_{i}\right)=\sqrt{m^{2}+\mathbf{p}_{\perp}^{2}+\left(\pi_{D}\left(t_{i}\right)\right)^{2}}$ is the energy of a particle in the initial and final time instants $t_{i}$ (the longitudinal momenta $\pi_{D}\left(t_{i}\right)=p_{D}-e A_{D}\left(t_{i}\right)$ in the T-constant field have the form $\pi_{D}\left( \pm \frac{T}{2}\right)=p_{D} \mp e E \frac{T}{2}$. Let us consider the variation of the total energy of the system, which goes over from the initial vacuum state $\mid 0$, in $>$ to the final state $\mid N$, out $>$ with pairs created in all the possible levels for the T-constant field,

$$
\mid N, \text { out }>=\prod_{\mathbf{p}_{\perp}, r,\left|p_{D}\right|<e E T / 2} a_{\mathbf{p}, r}^{\dagger}(\text { out }) b_{\mathbf{p}, r}^{\dagger}(\text { out }) \mid 0, \text { out }>.
$$

Then one can formally write the energy of the initial state as

$$
\mathcal{E}_{1}=-\sum_{\mathbf{p}, r} p_{0}\left(t_{1}\right)
$$

and of the final state as

$$
\mathcal{E}_{2}=\sum_{\mathbf{p}_{\perp}, r}\left[\sum_{\left|p_{D}\right|<e E T / 2} 2 p_{0}\left(t_{2}\right)-\sum_{p_{D}} p_{0}\left(t_{2}\right)\right] .
$$

Thus

$$
\Delta \mathcal{E}=\mathcal{E}_{2}-\mathcal{E}_{1}=\sum_{\mathbf{p}_{\perp}, r}\left(\sum_{\left|p_{D}\right|<e E T / 2}\left[p_{0}\left(t_{2}\right)+p_{0}\left(t_{1}\right)\right]+\Delta \mathcal{E}_{v a c}\right)
$$


where

$$
\Delta \mathcal{E}_{v a c}=\sum_{\left|p_{D}\right|>e E T / 2}\left[p_{0}\left(t_{1}\right)-p_{0}\left(t_{2}\right)\right]
$$

is the shift of the vacuum energy related to the levels with given $\mathbf{p}_{\perp}, r$, in which no pairs appear. We are going to analyze this shift only. That is why we do not discuss here regularization problems of the total sum (58) (that can be done, using, for example, the methods described in [11]). One can see that

$$
\sum_{\left|p_{D}\right|<e E T / 2}\left[p_{0}\left(t_{1}\right)-p_{0}\left(t_{2}\right)\right]=0
$$

since $p_{0}\left(t_{1}\right)-p_{0}\left(t_{2}\right)$ is an odd function of $p_{D}$. That allows one to extend the summation in (59) over all the longitudinal momenta. The vacuum before the time instant $t_{1}$ was free and therefore symmetric with respect to the longitudinal kinetic momentum $\pi_{D}\left(t_{1}\right)=\pi_{D}=$ $p_{D}+e E \frac{T}{2}$. Replacing the summation over $p_{D}$ by one over $\pi_{D}$, one can therefore treat the corresponding improper integral in sense of its principal value. Thus

$$
\begin{aligned}
\Delta \mathcal{E}_{v a c} & =\frac{L}{2 \pi} \lim _{M \rightarrow \infty} \int_{-M}^{M}\left(\sqrt{m^{2}+\mathbf{p}_{\perp}^{2}+\pi_{D}^{2}}-\sqrt{m^{2}+\mathbf{p}_{\perp}^{2}+\left(\pi_{D}-e E T\right)^{2}}\right) d \pi_{D} \\
& =-\frac{L}{2 \pi}(e E T)^{2}=-\frac{L}{2 \pi}\left[\pi_{D}\left(t_{2}\right)-\pi_{D}\left(t_{1}\right)\right]^{2} .
\end{aligned}
$$

Since the number of states with given $p_{D}$, in which particles can be created, is equal to $\frac{1}{2 \pi} e E L T$, see (35), then the shift (60) can be rewritten in the form

$$
\Delta \mathcal{E}_{v a c}=\sum_{\left|p_{D}\right|<e E T / 2} \Delta \epsilon_{v a c}, \Delta \epsilon_{v a c}=-e E T=-\left|\pi_{D}\left(t_{2}\right)-\pi_{D}\left(t_{1}\right)\right|
$$

Thus,

$$
\Delta \mathcal{E}=\sum_{\mathbf{p}_{\perp}, r} \sum_{\left|p_{D}\right|<e E T / 2} \Delta \epsilon, \Delta \epsilon=p_{0}\left(t_{2}\right)+p_{0}\left(t_{1}\right)+\Delta \epsilon_{v a c}
$$

where $\Delta \epsilon$ can be interpreted as a work which the external field accomplishes for the creation of a pair in a given state. It contains a contribution $\Delta \epsilon_{v a c}$ which takes into account a shift of the vacuum energy in those states which remain vacuum ones. The corresponding work with respect to a particle will be denoted by $\omega$, so that, 


$$
\begin{aligned}
\omega & =\frac{1}{2} \Delta \epsilon \\
& =\frac{1}{2}\left[\sqrt{m^{2}+\mathbf{p}_{\perp}^{2}+\left(\pi_{D}\left(t_{2}\right)\right)^{2}}+\sqrt{m^{2}+\mathbf{p}_{\perp}^{2}+\left(\pi_{D}\left(t_{1}\right)\right)^{2}}-\left|\pi_{D}\left(t_{2}\right)-\pi_{D}\left(t_{1}\right)\right|\right] .
\end{aligned}
$$

Now we remark that due to the conditions of the stabilization $T>>T_{0},\left|p_{D}\right|<e E \frac{T}{2}$, under which all the results for the T-constant field were obtained, we can write

$$
\omega=\frac{1}{4} \lambda e E\left(\frac{1}{\left|\pi_{D}\left(t_{2}\right)\right|}+\frac{1}{\left|\pi_{D}\left(t_{1}\right)\right|}\right)=\frac{\lambda}{T}=\frac{\lambda e E}{2 p_{0}\left(t_{2}\right)}, \quad \lambda=\frac{m^{2}+\mathbf{p}_{\perp}^{2}}{e E} .
$$

Then the spectrum (33) can be rewritten in the following form 1

$$
N_{\mathbf{p}, r}=\exp \left\{-2 \pi \frac{\omega}{\frac{\hbar}{c} g}\right\}
$$

where the quantity $g$ can be written in several equivalent forms

$$
g=\frac{c e E}{2}\left(\frac{1}{\left|\pi_{D}\left(t_{2}\right)\right|}+\frac{1}{\left|\pi_{D}\left(t_{1}\right)\right|}\right)=\frac{2 c}{T}=\frac{c e E}{p_{0}\left(t_{2}\right)} .
$$

The last expression in (66) allows one to treat $g$ as the classical acceleration of a particle in the electric field in the final time instant $t_{2}=\frac{T}{2}$, for the case when the action time of the field is big enough, so that the corresponding velocities are near $c$. Formally this is valid under the quantum condition of stabilization. The distribution (65) is, in fact, the Boltzmann one with the temperature $\theta=\frac{\hbar g}{2 \pi c k_{B}}$ having literally the Hawking form. Thus, if one identifies the work $\omega$, we have introduced, with the energy of a particle in the formula (55), then the distributions in electrodynamical and gravitational cases have the same thermal structure. Let us discuss now the possible origin of the differences in the electrodynamical and gravitational formulas. First of all, the formula (55) is derived in the formalism of the stationary scattering theory, where it is not necessary to take separately into account a shift of the vacuum level. In this case the energy of a particle created may coincide with the corresponding work of the field. Second, the different form of the thermal distributions (Boltzmann, Planck) can be stipulated by essentially different situations in

\footnotetext{
${ }^{1}$ we have restored $\hbar$ and $c$ here for convenience of the reader
} 
both cases. In the electrodynamical case we deal in fact with pure states, whereas in the gravitational problems a density matrix is arisen necessary due to the horizon of events formation. At $\omega / g<<1$ the Planck spectrum coincides with the Boltzmann one. In this case one can believe the form (65) for the spectrum of particles created is universal and applicable to any theory with quasiconstant external fields.

The form (65) can be useful to describe situations in constant fields, where it is convenient to avoid the consideration of the time evolution, as we have seen comparing it with the gravitational cases. Another form of the distribution (65) with $2 \omega=\Delta \epsilon$ from (63) (or (62)) and with the acceleration $g$ from (66), can be useful in problems with explicit time dependence.

The universality of the formula (65) can be examine also in the case of the adiabatic electric field, $\alpha>>\alpha_{0}$, considered in Sect.IIIB. To apply it to the latter case one needs to put $t_{2,1} \rightarrow \pm \infty$, then $\pi_{D}\left(t_{2}\right)=p_{D}-e E \alpha, \pi_{D}\left(t_{1}\right)=p_{D}+e E \alpha$ and $p_{0}\left(t_{2}\right)=\omega_{+}, p_{0}\left(t_{1}\right)=\omega_{-}$. In this case according to (61) $\Delta \epsilon_{\text {vac }}=-2 e E \alpha$ and the distribution (46) follows.

One can remark in this connection that in case $E / E_{c}<<1\left(\alpha_{0}<<T_{0}\right)$ the formulas (64) (and, therefore, (63)) $)$ in the adiabatic field are valid at the condition $t_{2}=-t_{1}=\frac{T}{2}>>\alpha_{0} \sim$ $T_{0}^{f}$, which are weaker than in the case of the T-constant field. That allows one to interpret the time $T_{0}^{f}$, which already had appeared before in Subsect.A as a pair formation time, from another point of view. Considering the formula (63), one can see that at $T>>T_{0}^{f}$ the $\Delta \epsilon$ is less than $2 \sqrt{m^{2}+\mathbf{p}^{2}}$, due to the essential contribution of the vacuum shift $\Delta \epsilon_{\text {vac }}$. That means the work of the external field to produce a pair is less than one, which could be expected from the perturbation theory, where no vacuum change is taken into account.

The consideration presented was made only for fermions. However, if one believes that the quantity $\Delta \epsilon_{\text {vac }}$ can be taken in the form (61) for bosons as well, then the distribution (65) holds also in the scalar case. A consistent analysis for charged boson is more complicated and needs to take into account possible condensate formation and its evolution in an external field (see, for example, [5, 40]).

Finally, we believe that the formulas derived and the time-dimensional analysis presented 
can be also useful to describe some collective effects in the framework of quantum field theory, for instance, to describe multiple particles creation by means of the external field approach [13,14] or in string models with external field [11,12,41].

\section{ACKNOWLEDGMENTS}

D. Gitman and S. Gavrilov thank Brazilian foundations CNPq and FAPESP respectively for support. Besides, S. Gavrilov thanks Russian Foundation of Fundamental Research which is supporting him in part under the Grant No 94-02-03234. Both thank Prof. J. Frenkel for useful discussions and friendly support. 


\section{REFERENCES}

[1] O. Klein, Z. Phys. 53, 157 (1929); F. Sauter, Z. Phys. 69, 742 (1931); F. Sauter, Z. Phys. 73, $547(1931)$

[2] J. Schwinger, Phys. Rev. 82, 664 (1951)

[3] A.I. Nikishov, in Quantum Electrodynamics of Phenomena in Intense Fields, Proc. P.N. Lebedev Phys. Inst. 111, 153 (Nauka, Moscow 1979)

[4] W. Greiner, B. Müller, and J. Rafelski, Quantum Electrodynamics of Strong Fields (Springer-Verlag, Berlin 1985)

[5] A.A. Grib, S.G. Mamaev, and V.M. Mostepanenko, Vacuum Quantum Effects in Strong Fields (Atomizdat, Moscow 1988; Friedmann Laboratory Publishing, St. Petersburg 1994)

[6] E.S. Fradkin, D.M. Gitman and S.M. Shvartsman, Quantum Electrodynamics with Unstable Vacuum (Springer-Verlag, Berlin 1991)

[7] N.D. Birrel and P.C.W. Davis, Quantum Fields in Curved Space (Cambridge University Press, Cambridge 1994)

[8] S.W. Hawking, Commun. Math. Phys. 43, 199 (1975); Phys. Rev. D14, 2460 (1976)

[9] S.P. Gavrilov and D.M. Gitman, Preprint MIT, Massachusetts, 1991, CTP\#1995; S.P. Gavrilov, Russian Phys. Journ. 35, No 7, 652 (1992); S.P. Gavrilov, ibid, 35, No 10, 969 (1992); S.P. Gavrilov, ibid, 36, No 3, 269 (1993); S.P. Gavrilov and D.M. Gitman, ibid, 36, No 5, 448 (1993); S.P. Gavrilov and D.M. Gitman, ibid (Izv. Vuzov), No 4, 83 (1995)

[10] J. Zinn-Justin, Quantum Field Theory and Critical Phenomena (Oxford University Press, Oxford 1993)

[11] E. Elizalde, S.D. Odintsov et al, Zeta Regularization Techniques with Applications 
(World Scientific, Singapore 1994)

[12] I.L. Buchbinder, S.D. Odintsov and I.L. Shapiro, Effective Action in Quantum Gravity (IOP Publishing, Bristol and Philadelphia 1992); S. Odintsov, Fortschr.Phys. 38, 371 (1990); ibid. 39, 621 (1991)

[13] I.M. Dremin and A.V. Leonidov, Usp. Fiz. Nauk 165, 759 (1995)

[14] Y. Kluger, J.M. Eisenberg, B. Svetitski, F. Cooper and E. Mottola, Phys. Rev. Lett. 67, 2427 (1991); Phys. Rev. D 45, 4659 (1992)

[15] C. Kiefer and A. Wipf, Ann. Phys. 236, 241 (1994)

[16] W. Sigel, Nucl. Phys. B156, 135 (1979); R. Jackiw and S. Templeton, Phys.Rev. D23, 2291 (1981); J.F. Schonfeld, Nucl. Phys. B185, 157 (1981); S. Deser, R. Jackiw, and S. Templeton, Ann. Phys. (N.Y.) 140, 372 (1982); 185, 406(E) (1988); S. Forte, Int. J. Mod. Phys.A 7, 1025 (1992)

[17] F. Wilczek, Fractional Statistics and Anyon Superconductivity (World Scientific Publishing, Singapore 1990)

[18] A.I. Nikishov, Sov. Phys. JETP 30, 660 (1970)

[19] D.M. Gitman, J. Phys. A 10, 2007 (1977);

[20] S.P. Gavrilov and D.M. Gitman, Sov. Phys. Journ. No. 6, 491 (1980)

[21] V.G. Bagrov and D.M. Gitman, Exact Solutions of Relativistic Wave Equations, (Kluwer, Dordrecht, Boston, London 1990)

[22] R. Brauer and H. Weyl, Amer. Journ. Math. 57, 425 (1935)

[23] V.G. Bagrov, D.M. Gitman and V.A. Kuchin, in Actual Problems of Theoretical Physics (MGU, Moscow 1976) 334

[24] S.P. Gavrilov and D.M. Gitman, Sov. J. Nucl. Phys. (USA) 51, 1040 (1990) 
[25] M.S. Marinov and V.S. Popov, Sov. J. Nucl. Phys. (USA) 15, 702 (1972)

[26] M.S. Marinov and V.S. Popov, Sov. J. Nucl. Phys. (USA) 16, 449 (1973)

[27] H. Bateman and A. Erdelyi, Higher Transcendental functions, Vol. 1,2 (McGraw-Hill, New York 1953)

[28] V.G. Bagrov, D.M. Gitman and Sh.M. Shvartsman, Sov. Phys. JETP 41, 191 (1975)

[29] J. Hallin and P. Liljenberg, Phys. Rev. D 52, 1150 (1995)

[30] N.B. Narozhny and A.I. Nikishov, Sov. J. Nucl. Phys. (USA) 11, 596 (1970)

[31] E.B. Brezin and C. Itzykson, Phys. Rev. D 2, 1191 (1970); V.S. Popov, Sov. J. Nucl. Phys. (USA) 19, 584 (1974)

[32] N.B. Narozhny and A.I. Nikishov, Sov. Phys. JETP 38, 427 (1974); V.M. Mostepanenko and V.M. Frolov, Sov. J. Nucl. Phys. (USA) 19, 451 (1974)

[33] N.B. Narozhny and A.I. Nikishov, Theor. Math. Phys. 26, 9 (1976)

[34] S.P. Gavrilov, D.M. Gitman and Sh.M. Shvartsman, Sov. J. Nucl. Phys. (USA) 29, 567 (1979); $715(1979)$

[35] S.A. Fulling, Phys. Rev. D7, 2850 (1973); P.C.W. Davies, J. Phys. A 8, 609 (1975); W.G. Unruh, Phys. Rev. D14, 870 (1976)

[36] V.M. Villalba, Phys. Rev. D52, 3742 (1995); S. Bisvas, J. Guha and N.G. Sarkar, Class. Quant. Grav. 12, 1591 (1995); J. Guha et al, ibid, 1641 (1995)

[37] V.I. Ritus, Sov. Phys. JETP 53, 659 (1981)

[38] B. Müller, W. Greiner and J. Rafelski, Phys. Lett. A63, 181 (1977)

[39] D.M. Gitman and V.P. Frolov, Sov. J. Nucl. Phys. 28, 281 (1978); V.P. Frolov and D.M. Gitman, J. Phys. A15, 1329 (1978) 
[40] A.B. Migdal, Fermions and Bosons in Strong Fields (Nauka, Moscow 1978); A.B. Migdal, Sov. Phys. JETP 34, 1184 (1971)

[41] H. Kanasugi, H. Okada, Progr. Theor. Phys. 79, 1197 (1988) 